\title{
Giá trị cảm nhận, chất lượng mối quan hệ và sự gắn kết của khách hàng trong bối cảnh dịch vụ nhà hàng
}

\section{Perceived value, relationship quality and customer engagement in restaurant service context}

\author{
Nguyễn Hữu Khôi ${ }^{1 *}$ \\ ${ }^{1}$ Trường Đại học Nha Trang, Việt Nam \\ *Tác giả liên hệ, Email: khoinh@ntu.edu.vn
}

THÔNG TIN

DOI: $10.46223 / \mathrm{HCMCOUJS.}$ econ.vi.17.1.1424.2022

Ngày nhận: 29/01/2021

Ngày nhận lại: 23/03/2021

Duyệt đăng: 01/04/2021

Tù khóa:

chất lượng quan hệ; dịch vụ nhà hang; gắn kết của khách hàng; giá trị cảm nhận

Keywords:

relationship quality; restaurant service; customer engagement; perceived value

\section{TÓM TÁT}

Nghiên cứu này nhằm mục đích thảo luận và kiểm định mối quan hệ giữa giá trị cảm nhận, chất lượng mối quan hệ và sự gắn của khách hàng trong bối cảnh dịch vụ nhà hàng tại Việt Nam. Theo đó, giá trị cảm nhận được giả thuyết có tác động trực tiếp và gián tiếp đến sự gắn kết của khách hàng thông qua chất lượng mối quan hệ. Kết quả kiểm định giả thuyết trên một mẫu 219 khách hàng tại Thành phố Hồ Chí Minh cho thấy rằng giá trị cảm nhận có tác động trực tiếp đến chất lượng mối quan hệ và sự gắn kết cũng như có tác động gián tiếp đến sự gắn kết thông qua chất lượng mối quan hệ. Từ kết quả nghiên cứu, chúng tôi đề xuất một vài hàm ý học thuật và thực tiễn cho hoạt động quản lý nhà hàng.

\section{ABSTRACT}

This study discusses and tests the relationships between perceived value, relationship quality, and customer engagement in the Vietnamese restaurant service context. Accordingly, perceived value is hypothesized to have a direct and indirect effect on customer engagement via relationship quality. The hypothesis testing results on a sample of 219 customers in Ho Chi Minh City indicate that perceived value directly affects relationship quality and customer engagement while also has an indirect effect on customer engagement via relationship quality. Based on the findings, we propose some theoretical and practical implications for restaurant management activities.

\section{Giới thiệu}

Chiến lược marketing của doanh nghiệp đã thay đổi từ tập trung vào giao dịch vào những năm 1990 đến phát triển chất lượng mối quan hệ vào những năm 2000 (Aurier \& N'Goala, 2009; Moliner, Sánchez, Rodríguez, \& Callarisa, 2007) và hiện nay là sự gắn kết của khách hàng (customer engagement) (Kumar, Rajan, Gupta, \& Pozza, 2019; Pansari \& Kumar, 2016). Dù vậy, quá trình nghiên cứu sự gắn kết của khách hàng chỉ đang ở giai đoạn bắt đầu và tập trung vào lập luận ở mức khái niệm (France, Merrilees, \& Miller, 2016). Thật vậy, các nghiên cứu trước đây về sự gắn kết thường tập trung vào xây dựng thang đo lường của sự gắn kết của khách 
hàng (Hollebeek, Glynn \& Brodie, 2014) và xây dựng các mô hình quan niệm gồm các tiền đề cho biến số này (Kumar et al., 2019; Pansari \& Kumar, 2016). Số lượng các nghiên cứu thực nghiệm kiểm định tác động của các tiền đề dẫn đến việc hình thành sự gắn kết của khách hàng vẫn còn hạn chế (France et al., 2016; Hollebeek et al., 2014). Vì vậy, các nghiên cứu trong quá khứ được thực hiện trong bối cảnh Việt Nam (Nguyen \& Le, 2019) và quốc tế (Harrigan, Evers, Miles, \& Daly, 2018; Roy, Shekhar, Lassar, \& Chen, 2018) đều khuyến nghị việc xây dựng các mô hình thực nghiệm giải thích sự gắn kết của khách hàng. Gần đây, lĩnh vực dịch vụ cũng nhấn mạnh vai trò của sự gắn kết của khách hàng và phát triển các mô hình giải thích sự gắn kết của khách hàng (Ahn \& Back, 2018; Roy, Shekhar, et al., 2018). Vì vậy, nghiên cứu này xây dựng mô hình thực nghiệm giải thích sự gắn kết của khách hàng trong bối cảnh dịch vụ nhà hàng tại Việt Nam.

Các nghiên cứu trước đây đã gợi ý mối liên kết giữa chất lượng mối quan hệ và sự gắn kết của khách hàng (Bowden, 2009b; Kumar et al., 2019; Pansari \& Kumar, 2016). Dựa trên khung lý thuyết này, Tonder và Petzer (2018) kiểm định mối quan hệ giữa chất lượng mối quan hệ và sự gắn kết trong bối cảnh dịch vụ bảo hiểm. Tuy nhiên, mối quan hệ giữa các biến số này chưa được kiểm định trong bối cảnh dịch vụ nhà hàng, đặc biệt tại Việt Nam. Vì vậy, câu hỏi: "liệu rằng hoạt động marketing quan hệ của nhà hàng có giúp gia tăng sự gắn kết và làm sao để cũng cố mối quan hệ với khách hàng?” trong bối cảnh này vẫn chưa được trả lời. Bên cạnh đó, Itani, Kassar, và Loureiro (2019) và Tonder và Petzer (2018) đề xuất rằng giá trị cảm nhận sẽ tác động đến chất lượng mối quan hệ, từ đó tác động đến sự gắn kết của họ đối với của khách hàng. Giá trị cảm nhận có vai trò quan trọng trong dịch vụ nhà hàng (Itani et al., 2019; Roy, Balaji, Soutar, Lassar, \& Roy, 2018; Tonder \& Petzer, 2018). Tuy nhiên, vai trò của biến số này đối với sự gắn kết của khách hàng trong bối cảnh dịch vụ nhà hàng vẫn chưa được khám phá. Vì vậy, nghiên cứu này tích hợp giá trị cảm nhận vào mô hình nghiên cứu nhằm kiểm định đóng góp trực tiếp của biến số này đến chất lượng mối quan hệ cũng như đóng góp gián tiếp vào sự gắn kết của khách hàng thông qua chất lượng mối quan hệ.

Tựu trung lại, thông qua việc thảo luận và kiểm định mô hình đề xuất, kết quả nghiên cứu này điền vào khoảng trống về kiến thức liên quan đến việc bằng cách nào sự gắn kết của khách hàng có thể được xây dựng thông qua marketing quan hệ (Bowden, 2009b; Kumar et al., 2019; Pansari \& Kumar, 2016) và giá trị cảm nhận (Itani et al., 2019; Roy, Balaji, et al., 2018; Tonder \& Petzer, 2018) trong lĩnh vực kinh doanh dịch vụ nhà hàng tại Việt Nam. Từ đó, các nhà quản trị nhà hàng có thể phát triển chiến lược marketing hiệu quả thúc đẩy sự gắn kết của khách hàng.

\section{Cơ sở lý thuyết và mô hình nghiên cứu}

\subsection{Sụ̂ gắn kết của khách hàng}

Nghiên cứu này định nghĩa sự gắn kết của khách hàng là một trạng thái tâm lý bắt nguồn từ những trải nghiệm tương tác và đồng sáng tạo với một của khách hàng mà họ có quan hệ thân thiết (Brodie, Hollebeek, Jurić, \& Ilić, 2011; Hollebeek, 2011b). Trạng thái gẳn kết có tính lâu dài và lan tỏa, được đặc trưng bởi mức độ đắm chìm và đam mê của khách hàng (Hollebeek, 2011b). Sự gắn kết của khách hàng gồm ba thành phần nhận thức, tình cảm và hành vi, phản ánh động cơ của khách hàng gắn liền với nhà hàng (Hollebeek, 2011a).

Một vài mô hình lý thuyết gợi ý rằng sự gắn kết là kết quả của chất lượng mối quan hệ giữa nhà hàng và khách hàng (Bowden, 2009b; Kumar et al., 2019; Pansari \& Kumar, 2016). Ví dụ, Bowden (2009b) lập luận rằng sự hài lòng của khách hàng sẽ giúp hình thành sự tin tưởng, sự cam kết và cuối cùng là sự trung thành của họ. Pansari và Kumar (2016) cho rằng hài lòng có đóng góp trực tiếp vào sự gắn kết của khách hàng và giá trị cảm nhận có tác động tích cực đến sự 
hài lòng của khách hàng. Tương tự, Itani và cộng sự (2019) và Tonder và Petzer (2018) thảo luận mối quan hệ giá trị cảm nhận, chất lượng mối quan hệ và sự cam kết trong bối cảnh dịch vụ. Như vậy, trong bối cảnh dịch vụ, giá trị cảm nhận có quan hệ trực tiếp với chất lượng mối quan hệ, từ đó gia tăng sự gắn kết của họ với nhà hàng. Hơn nữa, các nghiên cứu cũng cho thấy rằng sự gắn kết sẽ mang lại những kết quả tích cực cho doanh nghiệp (France et al., 2016). Vì vậy, việc khám phá thực nghiệm các nhân tố đóng góp vào sự gắn kết của khách hàng có vai trò quan trọng đối với hoạt động quản trị, đặc biệt trong lĩnh vực dịch vụ như dịch vụ nhà hàng (Ahn \& Back, 2018; Roy, Shekhar, et al., 2018).

Tuy nhiên, mối quan hệ giữa giá trị cảm nhận, chất lượng mối quan hệ và sự gắn kết dường như bị bỏ qua (Itani et al., 2019; Tonder \& Petzer, 2018), đặc biệt trong bối cảnh dịch vụ nhà hàng. Một nghiên cứu thực nghiệm thảo luận và kiểm định các mối quan hệ này giúp củng cố niềm tin rằng khi nhà hàng đầu từ vào giá trị cảm nhận, họ sẽ tạo được một mối quan hệ có chất lượng với khách hàng, từ đó sẽ có được sự gắn kết và đạt được giá trị trọn đời của khách hàng.

\subsection{Giá trị cảm nhận và chất lự̛ng mối quan hệ}

Giá trị cảm nhận được định nghĩa là đánh giá tổng thể về lợi ích của sản phẩm dựa trên cảm nhận về những gì nhận được và những gì mất đi (Zeithaml, 1988). Trong khi đó, các nghiên cứu trước đây lập luận rằng cùng với sự hài lòng, sự tin tường và sự cam kết ba thành phần quan trọng của chất lượng mối quan hệ (Aurier \& N'Goala, 2009; Garbarino \& Johnson, 1999; Morgan \& Hunt, 1994). Sự hài lòng là kết quả của việc so sánh giữa hoạt động thực tế của một nhà cung cấp dịch vụ và kì vọng của khách hàng (Nysveen, Oklevik, \& Pedersen, 2018). Sự tin tưởng hàm ý rằng khách hàng tin chắc về sự đáng tin cậy và sự chính trực của nhà cung cấp dịch vụ nhà hàng (Morgan \& Hunt, 1994) và sự cam kết được định nghĩa là việc khách hàng tin rằng mối quan hệ giữa họ với nhà hàng là quan trọng và xứng đáng nhận được sự nỗ lực hết mình đề duy trì mối quan hệ này (Aurier \& N'Goala, 2009; Garbarino \& Johnson, 1999; Morgan \& Hunt, 1994).

Các nghiên cứu trước đây nhấn mạnh vai trò của giá trị cảm nhận và lâp luận rằng doanh nghiệp cần mang đến nhiều lợi ích cho khách hàng hơn chi phí họ phải bỏ ra. Parasuraman và Grewal (2000) gợi ý rằng giá trị cảm nhận có tác động đến sự hài lòng vì những lợi ích mà khách hàng đạt được lớn hơn những chi phí mà họ bỏ ra. Tượng tự, Tonder và Petzer (2018) cũng thảo luận và chứng minh rằng giá trị cảm nhận có tác động đến sự tin tưởng vì khi họ cảm nhận được giá trị do doanh nghiệp mang lại lớn hơn đối thủ cạnh tranh, họ sẽ tin rằng doanh nghiệp có đủ năng lực để đáp ứng nhu cầu của họ. Tương tự, Vieira (2013) chứng minh rằng giá trị cảm nhận tác động đến hài lòng và sự cam kết của khách hàng. Cuối cùng, Itani và cộng sự (2019) lập luận rằng giá trị cảm nhận không chỉ tác động đến hành vi trước trước mua hàng, mà còn ảnh hưởng đến hành vi trong và sau khi mua hàng. Lập luận này hàm ý rằng giá trị cảm nhận tác động đến các thành phần của chất lượng mối quan hệ gồm sự hài lòng, sự tin tưởng và sự cam kết hệ (Aurier \& N'Goala, 2009; Garbarino \& Johnson, 1999; Morgan \& Hunt, 1994). Vì vậy, nghiên cứu này giả thuyết.

H1: Giá trị cảm nhận có tác động có tác động tích cực đến chất lượng mối quan hệ giữa khách hàng với nhà hàng

\subsection{Chất lự̛ng mối quan hệ và sụ gắn kết của khách hàng}

Hài lòng là niềm tin rằng họ (khách hàng) sẽ tiếp tục được hưởng thụ những lợi ích khi sử dụng dịch vụ của nhà cung cấp của một nhà cung cấp dịch vụ nào đó. Rõ ràng, khi khách hàng hài lòng về dịch vụ nhà hàng của nhà cung cấp, họ sẽ có khuynh hướng cao hơn trong việc tiếp tục sử dụng dịch vụ của nhà hàng đó trong những lần sau. Do đó, hài lòng là cơ sở và tiền đề của sự gắn 
kết (Brodie et al., 2011). Bên cạnh đó, khi khách hàng tin tưởng một nhà hàng, họ sẽ đề cao mối quan hệ giữa mình và nhà hàng đó cũng như thể hiện sự gắn kết (Brodie et al., 2011; Itani et al., 2019). Lập luận này phù hợp với lý thuyết trao đổi xã hội, rằng sự nghi ngờ sẽ làm giảm sự gắn kết trong một mối quan hệ và do đó, các giao dịch thực hiện giữa các bên thường là ngắn hạn (Morgan \& Hunt, 1994). Cuối cùng, Mollen và Wilson (2010) xem sự gắn kết của khách hàng được hình thành từ sự cam kết về mặt nhận thức và sự cam kết về mặt tình cảm. Quan điểm này cũng được sự ủng hộ của Bowden (2009b) trong mô hình lý thuyết về sự hình thành sự gắn kết, trong đó cam kết về mặt nhận thức và tình cảm là các nhân tố quan trọng của sự gắn kết. Bên cạnh đó, quản lý quan hệ khách hàng đã chuyển dịch từ việc đặt trọng tâm vào chất lượng mối quan hệ đến thúc đẩy sự gắn kết với doanh nghiệp trên mọi khía cạnh (Pansari \& Kumar, 2016) cho thấy chất lượng mối quan hệ là tiền đề của sự gắn kết (Doorn et al., 2010). Kết quả kiểm định thực nghiệm trong lĩnh vực dịch vụ cho thấy rằng quan hệ khách hàng là tiền đề của sự gắn kết (Itani et al., 2019; Tonder \& Petzer, 2018). Vì vậy, nghiên cứu này giả thuyết rằng:

H2: Chất luợng mối quan hệ có tác động có tác động tích cực đến sự gắn kết của khách hàng với nhà hàng

\subsection{Tác động của giá trị và đến sụ gắn kết của khách hàng}

Parasuraman và Grewal (2000) lập luận rằng giá trị cảm nhận không chỉ tác động đến chất lượng mối quan hệ (e.g., hài lòng) mà còn ảnh hưởng đến hoạt động sau mua hàng của khách hàng (e.g., khuyến nghị cho bạn bè, tiếp tục mua hàng). Trong khung lý thuyết của Bowden $(2009 \mathrm{a}, 2009 \mathrm{~b})$ về sự gắn kết của về khách hàng trong lĩnh vực dịch vụ, các tác giả này cho rằng sự gắn kết chịu ảnh hưởng mạnh của giá trị cảm nhận của khách hàng. Bên cạnh đó, lý thuyết về sự gắn kết của Pansari và Kumar (2016) và sự gắn kết trong lĩnh vực dịch vụ của Kumar và cộng sự (2019) đều nhấn mạnh rằng khách hàng sẽ gắn kết với doanh nghiệp nếu họ nhận được giá thấp hơn và nhận đượ lợi ích tối đa (chính là giá trị).

Tương tự, Brodie và cộng sự (2011) cho rằng giá trị cảm nhận làm gia tăng sự gắn kết của khách hàng. Cùng quan điểm, Brodie, Ilic, Juric, và Hollebeek (2013) lập luận rằng khách hàng có động lực gia tăng sự gắn kết khi với một doanh nghiệp khi doanh nghiệp đó cung cấp dịch vụ mang lại nhiều giá trị hơn đối thủ cạnh tranh. Kết quả thực nghiệm trong một số nghiên cứu (Itani et al., 2019; Liu \& Jang, 2009) cho thấy rằng giá trị cảm nhận có tác động đến sự gắn kết của khách hàng trong bối cảnh dịch vụ. Vì vậy nghiên cứu này giả thuyết rằng trong bối cảnh dịch vụ nhà hàng:

H3: Giá trị cảm nhận có tác động có tác động tích cực đến sụ gắn kết của khách hàng đối với nhà hàng

\subsection{Tác động gián tiếp của giá trị và đến sụ gắn kết của khách hàng thông qua chất lựng mối quan hệ}

Vì giá trị cảm nhận có thể có tác động đến chất lượng mối quan hệ với doanh nghiệp (Itani et al., 2019; Parasuraman \& Grewal, 2000; Tonder \& Petzer, 2018; Vieira, 2013) và chất lượng mối quan hệ có tác động đến sự gắn kết của khách hàng (Itani et al., 2019; Kumar et al., 2019; Liu \& Jang, 2009; Pansari \& Kumar, 2016), nghiên cứu này kì vọng rằng giá trị cảm nhận sẽ có tác động gián tiếp đến sự gắn kết của khách hàng trong lĩnh vực dịch vụ nhà hàng. Kỳ vọng này phù hợp với khung lý thuyết của Bowden (2009a, 2009b) rằng chất lượng mối quan hệ đóng vai trò trung gian kết nối giá trị cảm nhận và sự gắn kết của khách hàng. Kết quả thực nghiệm của Kim và Han (2008) cung cho thấy vai trò trung gian của chất lượng mối quan hệ giữa chất lượng cảm nhận và hành vi mua hàng lặp lại (chính là sự gắn kết). Do đó, nghiên cứu này giả thuyết: 
H4: Giá trị cảm nhận có tác động gián tiếp đến sụ gắn kết của khách hàng đối với nhà hàng thông qua giá trị cảm nhận

Mô hình nghiên cứu được trình bày như Hình 1.

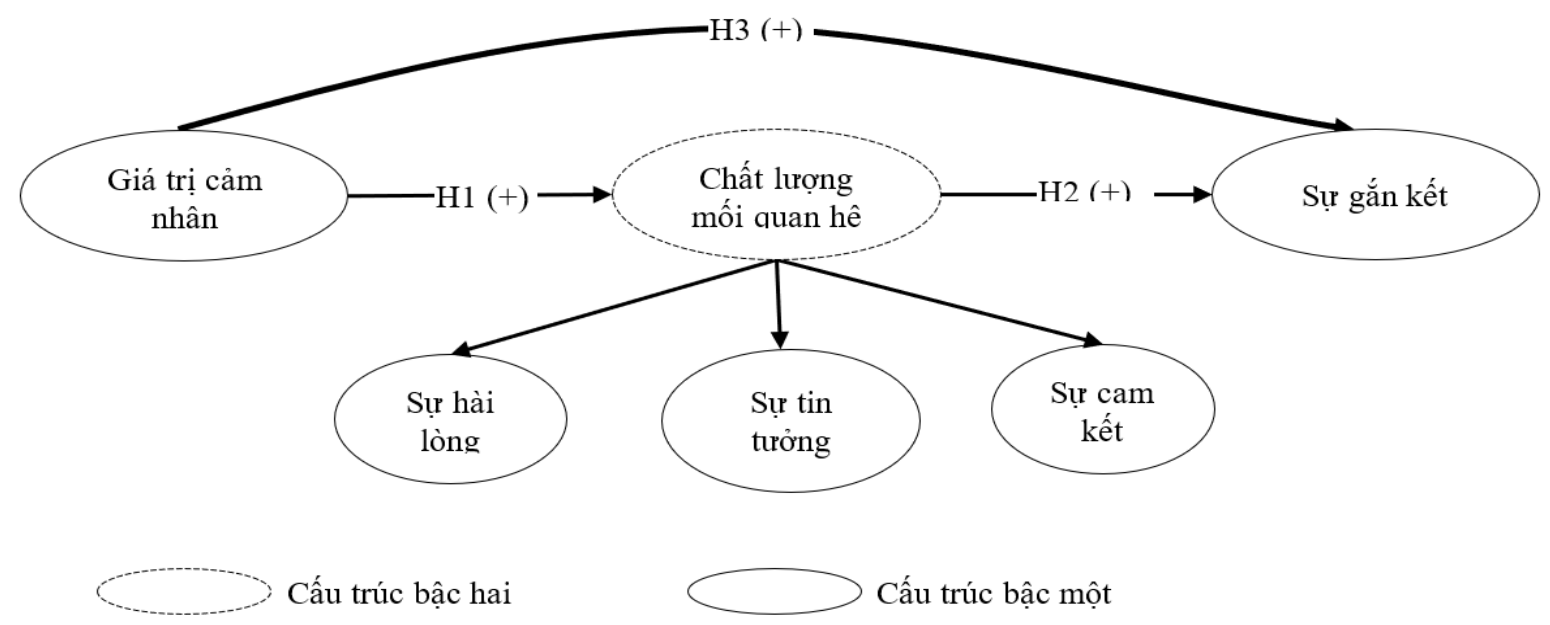

Hình 1. Mô hình và giả thuyết nghiên cứu

\section{Phương pháp nghiên cứu}

\subsection{Mẫu nghiên cứu}

Đối tượng thu mẫu trong nghiên cứu này là các khách hàng tại các nhà hàng tại Thành phố Hồ Chí Minh. Nghiên cứu này sử dụng phương pháp thu mẫu thuận tiện trong thời gian trong tháng 06 và 07 năm 2020. Các đáp viên là những người sống và làm việc tại Thành phố Hồ Chí Minh. Do đó, nghiên cứu đưa ra câu hỏi sàng lọc "Các $\mathrm{Anh} / \mathrm{Chị} \mathrm{có} \mathrm{đang} \mathrm{sống} \mathrm{và} \mathrm{làm} \mathrm{việc} \mathrm{tại}$ Thành phố Hồ Chí Minh không?” để loại trừ khách du lịch vì việc lựa chọn nhà hàng của du khách có thể chịu ảnh hưởng từ các yếu tố khác nhau (e.g., chịu tác động của hướng dẫn viên du lịch) và do đó có thể làm kết quả nghiên cứu bị chệnh. Các khách hàng trong nhà hàng được tiếp cận và được đề nghị tham gia nghiên cứu. Các khách hàng được đảm bảo thông tin thu thập được bảo mật và chỉ sử dụng cho mục đích nghiên cứu.

Tổng cộng có 230 khách hàng đồng ý tham gia khảo sát. Sau khi 230 phiếu khảo sát được thu lại, quá trình sàng lọc loại ra 11 bảng khảo sát do thiếu các thông tin cần thiết. Vì vậy, một mẫu nghiên cứu gồm 219 phiếu khảo sát được sử dụng cho quá trình phân tích. Đặc điểm mẫu khảo sát được mô tả trong Bảng 1 .

\section{Bảng 1}

Đặc điểm mẫu thu thập

\begin{tabular}{|l|l|c|c|}
\hline \multicolumn{2}{|c|}{ Đặc điểm } & Số lượng & Phần trăm \\
\hline \multirow{4}{*}{ Giới tính } & Nam & 70 & 32.0 \\
\cline { 2 - 4 } & Nữ & 146 & 66.7 \\
\cline { 2 - 4 } & Khác & 3 & 1.4 \\
\hline \multirow{3}{*}{ Tuổi } & Từ 25 trở xuống & 89 & 40.6 \\
\cline { 2 - 4 } & Lớn hơn 25 đến dưới 35 & 47 & 21.5 \\
\cline { 2 - 4 } & Lớn hơn 35 đến dưới 45 & 36 & 16.4 \\
\hline
\end{tabular}




\begin{tabular}{|l|l|c|c|}
\hline \multicolumn{2}{|c|}{ Đặc điểm } & Số lượng & Phần trăm \\
\hline \multirow{3}{*}{ Thu nhập } & Từ 45 & 47 & 21.5 \\
\hline & Dưới 10 triệu & 153 & 69.9 \\
\cline { 2 - 4 } & Từ 10 đến dưới 15 triệu & 53 & 24.2 \\
\cline { 2 - 4 } & Từ 15 triểu trở lên & 13 & 5.9 \\
\hline
\end{tabular}

Nguồn: Tính toán của tác giả dựa trên mẫu thu thập

\subsection{Thang đo lừ̀ng}

Thang đo lường được kế thừa từ các nghiên cứu trước đây (Bảng 2). Cụ thể hơn, thang đo giá trị cảm nhận gồm 04 mục hỏi được kế thừa từ nghiên cứu của Itani và cộng sự (2019). Chất lượng mối quan hệ là một cấu trúc bậc hai dạng phản ánh - phản ánh gồm ba thành phần là hài lòng, sự tin tưởng và sự cam kết. Thang đo sự hài lòng gồm 05 mục hỏi được lấy từ nghiên cứu của Klaus và Maklan (2013), thang đo sự tin tưởng gồm 04 mục hỏi được sử dụng từ Chaudhuri và Holbrook (2001) và thang đo sự cam kết gồm 04 mục hỏi từ nghiên cứu của Eisingerich và Rubera (2010). Cuối cùng thang đo sự gắn kết gồm 07 mục hỏi được kế thừa từ nghiên cứu của France và cộng sự (2016).

\section{Kết quả phân tích}

Phương pháp PLS-SEM với phần mềm SmartPLS 3.3.2 được sử dụng để kiểm mô hình đô lường và mô hình cấu trúc. Cụ thể hơn, nghiên cứu này kiểm định độ tin cậy, độ giá trị hội tụ và phân biệt, và các giả thuyết nghiên cứu.

\section{1. Độ tỉn cậy và độ giá trị của các đo lường}

Kết quả cho thấy các giá trị Cronbach's Alpha và độ tin cậy tổng hợp đều gần bằng hoặc lớn hơn giá trị 0.7 . Do đó, nghiên cứu này kết luận các thang đo lường đạt được độ tin cậy. Bên cạnh đó, các hệ số tải nhân tố đều lớn hơn 0.6 và phương sai trích của các cấu trúc khái niệm đều lớn hơn 0.5. Do đó, các thang đo đạt được giá trị hội tụ.

\section{Bảng 2}

Cấu trúc khái niệm, độ tin cậy và độ giá trị

\begin{tabular}{|c|c|c|c|c|}
\hline Cấu trúc khái niệm và mục hỏi & FL & Alpha & $\mathbf{C R}$ & AVE \\
\hline Giá trị cảm nhận (GT) & & 0.73 & 0.83 & 0.55 \\
\hline Tôi cảm thấy rằng ăn ở nhà hàng này là lựa chọn tốt & 0.78 & & & \\
\hline Tôi vẫn tiếp tục ăn ở nhà hàng này cho dù giá cả có tăng chút ít & 0.67 & & & \\
\hline Dịch vụ của nhà hàng xứng đáng với chi phí tôi phải bỏ ra & 0.82 & & & \\
\hline Nhà hàng có nhiều lựa chọn món ăn phù hợp với khách hàng & 0.70 & & & \\
\hline Sụ hài lòng $(H L)$ & & 0.75 & 0.83 & 0.50 \\
\hline Cảm tưởng của tôi về nhà hàng này rất tích cực & 0.60 & & & \\
\hline $\begin{array}{l}\text { Tôi cảm thấy thỏa mãn với mức độ đáp ứng mong muốn cá nhân của nhà } \\
\text { hàng này }\end{array}$ & 0.65 & & & \\
\hline Tổng thể, tôi hài lòng với dịch vụ do nhà hàng này cung cấp & 0.74 & & & \\
\hline Tôi hài lòng vì nhà hàng mang lại cho tôi những dịch vụ tốt nhất & 0.81 & & & \\
\hline Mức độ đáp ứng của dịch vụ nhà hàng này khiến tôi hài lòng & 0.72 & & & \\
\hline
\end{tabular}




\begin{tabular}{|l|c|c|c|c|}
\hline \multicolumn{1}{|c|}{ Cấu trúc khái niệm và mục hỏi } & FL & Alpha & CR & AVE \\
\hline Sụ tin tương (TT) & & 0.74 & 0.84 & 0.56 \\
\hline Tôi tin tưởng nhà hàng này & 0.80 & & & \\
\hline Tôi trông cậy vào nhà hàng này & 0.80 & & & \\
\hline Đây là một nhà hàng trung thực & 0.68 & & & \\
\hline Nhà hàng này đáng tin cậy & 0.71 & & \\
\hline Sự cam kết $(C K)$ & & 0.75 & 0.84 & 0.57 \\
\hline Tôi cảm thấy mình trung thành với nhà hàng này & 0.73 & & \\
\hline $\begin{array}{l}\text { Ngay cả khi nhà hàng này khó đặt chỗ hơn các nhà hàng khác, tôi vẫn sẽ sử } \\
\text { dụng nhà hàng này }\end{array}$ & 0.79 & & & \\
\hline Tôi sẵn lòng nỗ lực nhiều hơn để vẫn là khách hàng của nhà hàng này & 0.73 & & & \\
\hline Tôi sẽ không sử dụng nhà hàng này trong tương lai (R) & 0.77 & & & \\
\hline Sự gắn kết (GK) & & 0.85 & 0.89 & 0.53 \\
\hline Khi sử dụng nhà hàng này, tôi thực sự cảm thấy gắn kết & 0.78 & & \\
\hline Tôi say mê nhà hàng này & 0.76 & & & \\
\hline Tôi cảm giác mình thuộc về nhà hàng này & 0.76 & & & \\
\hline Tôi có sự nhiệt tình đối với nhà hàng này & 0.67 & & \\
\hline $\begin{array}{l}\text { Khi sử dụng nhà hàng này, tâm trí tôi chỉ tập trung vào những gì đang diễn } \\
\text { ra tại nhàng }\end{array}$ & 0.69 & & & \\
\hline Khi tương tác với nhà hàng này, tôi tập trung hoàn toàn vào nhà hàng & 0.76 & & & \\
\hline Tôi là một khách hàng trung thành của nhà hàng này & 0.65 & & & \\
\hline Chất luợng mối quan hệ (CLQH) & & 0.69 & 0.83 & 0.61 \\
\hline Hài lòng & 0.79 & & & \\
\hline Tin tưởng & & & \\
\hline Cam kết & & & \\
\hline
\end{tabular}

Ghi chú: FL: hệ số tải; Alpha: Cronbach’s Alpha; CR: Độ tin cậy tổng hợp; AVE: Phương sai trích Nguồn: Tính toán của tác giả dựa trên mẫu thu thập

Nghiên cứu này xem xét độ giá trị phân biệt giữa các cấu trúc khái niệm dựa vào giá trị của ma trận HTMT. Theo đó, vì các giá trị tương quan trong ma trận HTMT đều nhỏ hơn 0.85 nên nghiên cứu này kết luận các cấu trúc khái niệm đạt được độ giá trị phân biệt.

\section{Bảng 3}

Tương quan giữa các cấu trúc khái niệm

\begin{tabular}{|c|c|c|c|c|c|c|}
\hline Biến số & $\mathbf{1}$ & $\mathbf{2}$ & $\mathbf{3}$ & $\mathbf{4}$ & $\mathbf{5}$ & $\mathbf{6}$ \\
\hline 1.GT & & & & & & \\
\hline 2.HL & 0.57 & & & & & \\
\hline 3.TT & 0.45 & 0.59 & & & & \\
\hline 4.CK & 0.42 & 0.50 & 0.59 & & & \\
\hline 5.GK & 0.60 & 0.40 & 0.38 & 0.50 & & \\
\hline 6.CLQH & 0.64 & N/A & N/A & N/A & 0.56 & \\
\hline
\end{tabular}

Nguồn: Tính toán của tác giả dựa trên mẫu thu thập 


\subsection{Kiểm định giả thuyết}

Kết quả kiểm định giả thuyết (Bảng 4) cho thấy các tác động trực tiếp đều có ý nghĩa thống kê. Cụ thể hơn, giá trị cảm nhận có tác động có ý nghĩa đến chất lượng mối quan hệ ( $\beta=$ $0.47 ; \mathrm{p}<0.001)$ và chất lượng mối quan hệ có tác động đến sự gắn kết của khách hàng $(\beta=0.24$; $\mathrm{p}<0.01)$. Cuối cùng, giá trị cảm nhận có tác động tích cực sự gắn kết $(\beta=0.40 ; \mathrm{p}<0.001)$.

Kết quả kiểm trị có tác động gián tiếp đến sự gắn kết thông qua chất lượng quan định tác động gián tiếp cho thấy giá hệ $(\beta=0.11 ; \mathrm{p}<0.05)$.

\section{Bảng 4}

Kết quả kiểm định mô hình nghiên cứu

\begin{tabular}{|c|c|c|c|c|c|c|}
\hline \multirow{2}{*}{ Đường dẫn } & \multirow{2}{*}{ Giả thuyết } & \multicolumn{3}{|c|}{ Mô hình nghiên cứu } & \multirow{2}{*}{ VIF } & \multirow{2}{*}{ Kết luận } \\
\hline & & Std. $\beta$ & $t$-value & Bootstrap & & \\
\hline \multicolumn{7}{|l|}{ Tác động trục tiếp } \\
\hline Giá trị $\rightarrow$ Chất lượng quan hệ & $\mathrm{H} 1$ & 0.47 & $6.71 * * *$ & {$[0.31 ; 0.59]$} & 1.00 & Ủng hộ \\
\hline Chất lượng quan hệ $\rightarrow$ Gắn kết & $\mathrm{H} 2$ & 0.24 & $2.8 * *$ & {$[0.09 ; 0.43]$} & 1.31 & Ủng hộ \\
\hline Giá trị $\rightarrow$ Gắn kết & $\mathrm{H} 3$ & 0.40 & $5.19 * * *$ & {$[0.22 ; 0.53]$} & 1.31 & Ủng hộ \\
\hline \multicolumn{7}{|l|}{ Tác động gián tiếp } \\
\hline Giá trị $\rightarrow$ Chất lượng quan hệ $\rightarrow$ Gắn kết & $\mathrm{H} 4$ & 0.11 & $2.35^{*}$ & {$[0.24 ; 0.22]$} & & Ủng hộ \\
\hline $\mathrm{R}^{2}$ & \multicolumn{6}{|c|}{$\mathrm{R}_{\text {Gắn kết }=0.32}$} \\
\hline Độ lớn tác động (f²) & \multicolumn{6}{|c|}{$\begin{array}{l}\mathrm{f}^{2} \text { Giá trị } \rightarrow \text { Chất lượng quan hệ }=0.28 ; \mathrm{f}^{2} \text { Chất lượng quan hệ } \rightarrow \text { Gắn kết }=0.07 ; \\
\mathrm{f}^{2}{ }_{\text {Giá trị } \rightarrow \text { Gắn kết }=0.18 ;}\end{array}$} \\
\hline Stone-Geisser's $Q^{2}$ & \multicolumn{6}{|c|}{$\mathrm{Q}^{2}$ Gắn kết $=0.15$} \\
\hline
\end{tabular}

Ghi chú: *** $\mathrm{p}<0.001 ; * * \mathrm{p}<0.01 ; * \mathrm{p}<0.05 ;$ ns không có ý nghĩa thống kê

Nguồn: Tính toán của tác giả dựa trên mẫu thu thập

Kiểm định bootstrap cho thấy các tác động có ý nghĩa đều có khoảng giá trị không chứa giá trị 0 . Vì vậy các kết quả kiểm định đáng tin cậy. Hơn nữa, kết quả cũng cho thấy mô hình giải thích được $32 \%$ biến thiên của sự gắn kết và chỉ số Stone-Geisser's $\mathrm{Q}^{2}>0$ cho thấy mô hình phù hợp với dữ liệu thu thập.

\section{Kết luận và đề xuất}

Sự gắn kết của khách hàng ngày càng được quan tâm của học giả (Brodie et al., 2011; Kumar et al., 2019; Pansari \& Kumar, 2016). Tuy nhiên, số lượng các nghiên cứu thực nghiệm thảo luận và kiểm định mô hình các tiền đề của sự gắn kết của khách hàng vẫn còn hạn chế (France et al., 2016). Cụ thể hơn, mối quan hệ giữa giá trị cảm nhận, chất lượng mối quan hệ và sự gắn kết (Itani et al., 2019; Tonder \& Petzer, 2018) chưa được kiểm định trong bối cảnh dịch vụ nhà hàng tại Việt Nam. Nghiên cứu này điền vào khoảng trống nói trên bằng việc phát triển và kiểm định một mô hình mối quan hệ giữa giá trị cảm nhận, chất lượng mối quan hệ (hài lòng, tin tưởng và cam kết) và sự gắn kết với dịch vụ nhà hàng. Ngoài các tác động trực tiếp, nghiên cứu còn đóng góp bằng việc phát hiện cơ chế tác động gián tiếp giữa giá trị cảm nhận và sự gắn kết thông qua chất lượng mối quan hệ. Vì vậy, nghiên cứu có một số đóng góp sau về mặt lý thuyết và quản trị. 


\subsection{Hàm ýlý thuyết}

Trước tiên, nghiên cứu này đóng góp vào việc khẳng định mối quan hệ giữa giá trị cảm nhận, chất lượng mối quan hệ và sự gắn kết của khách hàng trong bối cảnh dịch vụ nhà hàng. Kết quả nghiên cứu cho thấy giá trị cảm nhận có tác động đến chất lượng mối quan hệ. Như vậy, khi khách hàng cảm thấy những lợi ích mà đạt được lớn hơn những chi phí mà họ bỏ ra, họ sẽ cảm thấy hài lòng với dịch vụ nhà hàng (Parasuraman \& Grewal, 2000), cảm thấy tin tưởng vào năng lực của nhà hàng trong việc mang lại giá trị cho họ nhiều hơn đối thủ cạnh tranh (Tonder \& Petzer, 2018) cũng như cho thấy rằng giá trị cảm nhận làm gia tăng sự cam kết của khách hàng đối với dịch vụ nhà hàng (Vieira, 2013). Như vậy, giá trị cảm nhận đóng vai trò quan trọng trong việc xây dựng chất lượng mối quan hệ giữa nhà hàng và khách hàng. Kết quả này phù hợp với kết quả thực nghiệm của Itani và cộng sự (2019) rằng giá trị cảm nhận có tác động đến chất lượng quan hệ.

Kết quả nghiên cứu cũng cho thấy khi khách hàng cảm thấy mối quan hệ giữa họ và một nhà hàng là quan trọng và xứng đáng cho các nỗ lực để duy trì mối quan hệ này (chất lượng mối quan hệ), họ sẽ có khuynh hướng gắn kết với nhà hàng đó. Những khách hàng có chất lượng mối quan hệ tốt với nhà hàng, họ sẽ có khuynh hướng sử dụng dịch vụ nhiều hơn và lâu hơn, có thể tác động đến những người khác sử dụng dịch vụ bằng sự đam mê và nhiệt huyết của họ đối với nhà hàng. Kết quả này cũng phù hợp với mô hình lý thuyết của Bowden (2009a, 2009b), trong đó chất lượng mối quan hệ là tiền đề của sự gắn kết của khách hàng.

Đáng chú ý, giá trị cảm nhận có tác động trực tiếp và gián tiếp đến sự gắn kết của khách hàng thông qua chất lượng mối quan hệ. Cụ thể hơn, tác động trực tiếp cho thấy rằng giá trị cảm nhận không chỉ tác động đến chất lượng mối quan hệ mà còn ảnh hưởng đến sự gắn kết của khách hàng (Parasuraman \& Grewal, 2000). Kết quả này phù hơp với lập luận của Bowden (2009a, 2009b) rằng sự gắn kết chịu ảnh hưởng mạnh của giá trị cảm nhận của khách hàng và lý thuyết về sự gắn kết của (Kumar et al., 2019; Pansari \& Kumar, 2016) rằng khách hàng sẽ gắn kết với doanh nghiệp nếu họ nhận được giá thấp hơn và nhận được lợi ích tối đa. Kết quả thực nghiệm cũng chứng minh rằng giá trị cảm nhận có tác động đến sự gắn kết của khách hàng trong bối cảnh dịch vụ (Itani et al., 2019; Liu \& Jang, 2009). Vì vậy, kết quả nghiên cứu có sự tương đồng với các nghiên cứu trước đây.

Kết quả nghiên cứu cho thấy giá trị cảm nhận có tác động đến sự gắn kết thông qua chất lượng mối quan hệ. Mặc dù khung lý thuyết của Bowden (2009a, 2009b) lập luân rằng chất lượng mối quan hệ đóng vai trò trung gian kết nối giá trị cảm nhận và sự gắn kết của khách hàng, tác động gián tiếp của giá trị cảm nhận đến sự gắn kết thông qua chất lượng quan hệ vẫn chưa được khám phá. Kết quả thực nghiệm của Kim và Han (2008) cung cho thấy vai trò trung gian của chất lượng mối quan hệ giữa chất lượng cảm nhận và hành vi mua hàng lặp lại. Vì vậy, kết quả nghiên cứu giúp làm rõ cơ chế nếu như và bằng cách nào giá trị cảm nhận làm gia tăng sự gắn kết của khách hàng.

Cuối cùng, các nghiên cứu trước đây đã nhấn mạnh vai trò quan trọng của sự gắn kết thương hiệu trong bối cảnh dịch vụ (Bowden, 2009b; Kumar et al., 2019; Pansari \& Kumar, 2016), bao gồm cả bối cảnh nhà hàng (Bowden, 2009a; Itani et al., 2019). Điều này cũng được thể hiện thông qua xu hương chuyển dịch từ marketing giao dịch sang marketing quan hệ và hiện nay là marketing sự gắn kết (Kumar et al., 2019; Pansari \& Kumar, 2016). Vì vậy, các học giả luôn mong muốn tìm ra các yếu tố tác động đến sự gắn kết của khách hàng trong lĩnh vực dịch vụ, đặc biệt là bối cảnh nhà hàng. Do đó, thông qua việc kiểm định tác động trực tiếp của giá trị cảm nhận và chất lượng mối quan hệ cũng như tác động gián tiếp của giá trị cảm nhận đến sự gắn kết, nghiên cứu này đóng góp trong việc cũng cố lý thuyết liên quan đến sự gắn kết cũng như 
cung cấp bằng chức thực nghiệm về vai trò quan trọng của giá trị cảm nhận và chất lượng mối quan hệ trong việc xây dựng sự gắn kết của khách hàng.

\subsection{Hàm ý quản trị}

Kết quả nghiên cứu đã cho thấy rằng các nhà quản trị dịch vụ nhà hàng cần củng cố mối quan hệ với khách hàng để đạt được sự gắn kết. Hơn nữa, kết quả nghiên cứu cũng chỉ ra rằng, nhà quản trị có thể củng cố mối quan hệ thông qua việc tạo ra những giá trị cho khách hàng. Cụ thể hơn, giá trị cảm nhận không những là tiền đề tạo ra và củng cố chất lượng mối quan hệ, từ đó hình thành sự gắn kết, mà còn có tác động gián tiếp đến sự gắn kết. Vì vậy, chiến lược của nhà hàng tập trung vào việc tạo ra nhiều giá trị và xây dựng mối qua hệ với khách hàng đóng vai trò quan trọng trong việc gia tăng sự gắn kết. Hơn thế nữa, việc xây dựng mối quan hệ với khách hàng được xem là một hoạt động dài hạn vì sự tin tưởng và cam kết chỉ có thể đạt được trong một thời gian dài. Vì vậy, nhà quản lý cần tạo ra chiến lược tập trung vào giá trị khi chuẩn bị thực đơn, chất lượng dịch vụ và quản trị mối quan hệ với khách hàng vì hầu hết khách hàng sử dụng dịch vụ nhà hàng đều quan tâm đến giá trị mà họ nhận được. Các nhà quản trị cũng cần lưu ý rằng, việc phục các thực khách đề cao giá trị đồng thời cũng đòi hỏi nhiều nguồn lực hơn như xây dựng khung cảnh nhà hàng thích hợp với đối tượng khách hàng mục tiêu, đào tạo đội ngũ nhân viên chuyên nghiệp, thân thiện và linh hoạt trong xử lý sự cố với khách hàng. Do đó, họ cần đánh giá chi tiết những lợi ích đạt được khi phục vụ các thực khách này.

Một hàm ý khác rút ra từ kết quả nghiên cứu đó là nhà hàng có thể phân khúc khách hàng dựa trên mức độ sự gắn kế của họ. Nói cách khác, quá trình phân khúc, lựa chọn mục tiêu, định vị và khác biệt hóa có thể dựa vào sức mạnh của sử gắn kết của khách hàng với nhà hàng. Theo đó, người quản lý có thể thực thi các chiến lược gắn kết khác nhau thông qua việc xây dựng mối quan hệ bền vững bằng việc tạo ra các lợi ích tăng thêm vượt qua các chi phí mà khách hàng bỏ ra theo từng nhóm phân khúc khách hàng. Ví dụ, để gia tăng sự hài lòng, nhà hàng có thể cung cấp dịch vụ vượt qua kì vọng khách hàng, làm cho họ cảm nhận rằng giá trị họ đạt được hơn cả những gì họ kì vọng. Như vậy, nhà hàng cần hiểu rõ kì vọng của các khách hàng mục tiêu trong từng phần khúc và có kế hoạch tạo phục vụ cụ thể cho những khách hàng này. Để gia tăng sự tin tưởng, nhà hàng cần bằng mọi cách thực hiện được những cam kết của mình về dịch vụ với khách hàng đối với từng phân khúc. Thông qua đó, khách hàng sẽ hiểu rằng nhà hàng có đủ năng lực để thực hiện công việc của mình cũng như luôn hướng đến sự rõ ràng, ngay thẳng, chính trực trong hoạt động cung cấp dịch vụ. Nhà hàng sẽ mất đi lợi thế cạnh tranh khi không phân khúc khách hàng, không hiểu rõ khách hàng về khía cạnh gắn kết và không đáp ứng được giá trị kì vọng của khách hàng, từ đó ảnh hương đến chất lượng mối quan hệ và cuối cùng là sự gắn kết. Thông qua các hoạt động phân khúc và áp dụng các chiến lược marketing khác nhau liên quan đến giá trị, giá trị cảm nhận của thực khách của từng phân khúc sẽ tăng lên, kéo theo một mối quan hệ chất lượng và sự gắn kết.

\section{Tài liệu tham khảo}

Ahn, J., \& Back, K.-J. (2018). Antecedents and consequences of customer brand engagement in integrated resorts. International Journal of Hospitality Management, 75, 144-152.

Aurier, P., \& N'Goala, G. (2009). The differing and mediating roles of trust and relationship commitment in service relationship maintenance and development. Journal of the Academy of Marketing Science, 38(3), 303-325.

Bowden, J. (2009a). Customer engagement: A framework for assessing customer-brand relationships: The case of the restaurant industry. Journal of Hospitality Marketing \& Management, 18(6), 574-596. 
Bowden, J. (2009b). The process of customer engagement: A conceptual framework. Journal of Marketing Theory and Practice, 17(1), 63-74.

Brodie, R. J., Hollebeek, L. D., Jurić, B., \& Ilić, A. (2011). Customer engagement: Conceptual domain, fundamental propositions, and implications for research. Journal of Service Research, 14(3), 252-271.

Brodie, R. J., Ilic, A., Juric, B., \& Hollebeek, L. (2013). Consumer engagement in a virtual brand community: An exploratory analysis. Journal of Business Research, 66(1), 105-114.

Chaudhuri, A., \& Holbrook, M. B. (2001). The chain of effects from brand trust and brand affect to brand performance: The role of brand loyalty. Journal of Marketing, 65(2), 81-93.

Doorn, J. V., Lemon, K. N., Mittal, V., Nass, S., Pick, D., Pirner, P., \& Verhoef, P. C. (2010). Customer engagement behavior: Theoretical foundations and research directions. Journal of Service Research, 13(3), 253-266.

Eisingerich, A. B., \& Rubera, G. (2010). Drivers of brand commitment: A cross-national investigation. Journal of International Marketing, 18(2), 64-79.

France, C., Merrilees, B., \& Miller, D. (2016). An integrated model of customer-brand engagement: Drivers and consequences. Journal of Brand Management, 23(2), 119-136.

Garbarino, E., \& Johnson, M. S. (1999). The different roles of satisfaction, trust, and commitment in customer relationships. Journal of Marketing, 63(2), 70-87.

Harrigan, P., Evers, U., Miles, M. P., \& Daly, T. (2018). Customer engagement and the relationship between involvement, engagement, self-brand connection and brand usage intent. Journal of Business Research, 88(July), 388-396.

Hollebeek, L. D. (2011a). Demystifying customer brand engagement: Exploring the loyalty nexus. Journal of Marketing Management, 27(7/8), 785-807.

Hollebeek, L. D. (2011b). Exploring customer brand engagement: Definition and themes. Journal of Strategic Marketing, 19(7), 555-573.

Hollebeek, L. D., Glynn, M. S., \& Brodie, R. J. (2014). Consumer brand engagement in social media: Conceptualization, scale development and validation. Journal of Interactive Marketing, 28(2), 149-165.

Itani, O. S., Kassar, A.-N., \& Loureiro, S. M. C. (2019). Value get, value give: The relationships among perceived value, relationship quality, customer engagement, and value consciousness. International Journal of Hospitality Management, 80, 78-90.

Kim, W., \& Han, H. (2008). Determinants of restaurant customers' loyalty intentions: A mediating effect of relationship quality. Journal of Quality Assurance in Hospitality \& Tourism, 9(3), 219-239.

Klaus, P., \& Maklan, S. (2013). Towards a better measure of customer experience. International Journal of Market Research, 55(2), 227-246.

Kumar, V., Rajan, B., Gupta, S., \& Pozza, I. D. (2019). Customer engagement in service. Journal of the Academy of Marketing Science, 47(1), 138-160.

Liu, Y., \& Jang, S. (2009). Perceptions of Chinese restaurants in the U.S.: What affects customer satisfaction and behavioral intentions? International Journal of Hospitality Management, 28(3), 338-348. 
Moliner, M. A., Sánchez, J., Rodríguez, R. M., \& Callarisa, L. (2007). Relationship quality with a travel agency: The influence of the postpurchase perceived value of a tourism package. Tourism and Hospitality Research, 7(3/4), 194-211.

Mollen, A., \& Wilson, H. (2010). Engagement, telepresence and interactivity in online consumer experience: Reconciling scholastic and managerial perspectives. Journal of Business Research, 63(9/10), 919-925.

Morgan, R. M., \& Hunt, S. D. (1994). The commitment-trust theory of relationship marketing. Journal of Marketing, 58(3), 20-38.

Nguyen, K. H., \& Le, H. N. (2019). Mối quan hệ giữa trải nghiệm, sự cam kết và gắn kết thương hiệu: Vai trò trung gian và điều tiết của tham gia thương hiệu [The relationship between brand experience, brand commitment and brand engagement: The mediating and moderating role of brand involvement]. Tạp chí Kinh tế và Phát triển, 277(7), 63-72.

Nysveen, H., Oklevik, O., \& Pedersen, P. E. (2018). Brand satisfaction: Exploring the role of innovativeness, green image and experience in the hotel sector. International Journal of Contemporary Hospitality Management, 30(9), 2908-2924.

Pansari, A., \& Kumar, V. (2016). Customer engagement: The construct, antecedents, and consequences. Journal of the Academy of Marketing Science, 45(3), 294-311.

Parasuraman, A., \& Grewal, D. (2000). The impact of technology on the quality-value-loyalty chain: A research agenda. Journal of the Academy of Marketing Science, 28(1), 168-174.

Roy, S. K., Balaji, M. S., Soutar, G., Lassar, W. M., \& Roy, R. (2018). Customer engagement behavior in individualistic and collectivistic markets. Journal of Business Research, 86, 281-290.

Roy, S. K., Shekhar, V., Lassar, W. M., \& Chen, T. (2018). Customer engagement behaviors: The role of service convenience, fairness and quality. Journal of Retailing and Consumer Services, 44, 293-304.

Tonder, E. V., \& Petzer, D. J. (2018). The interrelationships between relationship marketing constructs and customer engagement dimensions. The Service Industries Journal, 38(13/14), 948-973.

Vieira, V. A. (2013). Antecedents and consequences of perceived value: A meta-analytical perspective. Journal of Customer Behaviour, 12(2), 111-133.

Zeithaml, V. A. (1988). Consumer perceptions of price, quality, and value: A means-end model and synthesis of evidence. Journal of Marketing, 52(3), 2-22. 